\title{
Long non-coding RNA GAS5 promotes natural killer cell cytotoxicity against gastric cancer by regulating miR-18a
}

\author{
M. F. WEI ${ }^{1,2}$, Z. S. GU' ${ }^{1}$ L. L. ZHENG ${ }^{1}$, M. X. ZHAO ${ }^{1}$, X. J. WANG ${ }^{1, \star}$ \\ ${ }^{1}$ Department of Integrated TCM \& Western Medicine, The First Affiliated Hospital of Zhengzhou University, Zhengzhou, Henan, China; \\ ${ }^{2}$ Department of Integrated TCM \& Western Medicine, The Academy of Medical Sciences of Zhengzhou University, Zhengzhou, Henan, China
}

*Correspondence: Wangxinjie1137@163.com

Received October 14, 2019 / Accepted January 22, 2020

\begin{abstract}
Natural killer (NK) cells play significant roles in spontaneous antitumor response in multiple cancers, including gastric cancer. Currently, lncRNAs were identified as essential modulators in the development of NK cells via competing for the target miRNA. However, the regulatory mechanism of GAS5 in NK cells remains largely elusive. The expressions of GAS5 and miR-18a in NK cells were measured by qRT-PCR. The killing effects of NK cells were conducted by lactate dehydrogenase (LDH) assay. Detection of IFN- $\gamma$ and TNF- $\alpha$ level was carried out using ELISA assay. The interaction between GAS5 and miR-18a was determined by the luciferase reporter system and RIP assay, respectively. We found that GAS5 expression was downregulated while miR-18a expression was upregulated in primary NK cells isolated from GC patient compared with the healthy controls. Moreover, activation of NK cells stimulated by IL- 2 enhanced the secretion of IFN- $\gamma$, TNF- $\alpha$, and the expression of GAS5. The deficiency of GAS5 significantly suppressed the secretion of IFN- $\gamma$ and TNF- $\alpha$ as well as the killing effect of NK cells. Subsequently, luciferase reporter and RIP assay confirmed the interaction between GAS5 and miR-18a. In addition, miR-18a inhibitor attenuated GAS5 silencing induced inhibition of the cytotoxicity of activated NK cells. In conclusion, GAS5 promotes the killing effect of the natural killer cells against GC by regulating miR-18a, providing promising strategies for NK cells based antitumor therapies.
\end{abstract}

Key words: GAS5, miR-18a, NK cell cytotoxicity, gastric cancer

Gastric cancer (GC) is malignant cancer which ranks as the second leading cause of cancer-related deaths globally [1]. Currently, surgical excision is considered as the most effective treatment for GC patients and postoperative immune response improves the therapeutic outcomes [2, 3]. Natural killer (NK) cells refer to innate immune cells with cytotoxic functions and cytokine secretion to kill virallyinfected and transformed cells [4]. Generally, they participate in spontaneous antitumor response in multiple cancers, such as pancreatic carcinoma, hepatocarcinoma, and breast cancer [5-7]. Various signals are involved in the activation of NK cells, such as interleukin-2 (IL-2), IL-12, IL-15, and IL-18. In particular, IL-2 stimulates the activation of $\mathrm{NK}$ cells and the release of cytokines and chemokines, including interferon- $\gamma$ (IFN- $\gamma$ ) and tumor necrosis factor alpha (TNF-a) [8]. However, the biological mechanism of NK cells in GC remains controversial.

Long non-coding RNAs (lncRNAs) are a class of RNAs more than 200 nucleotides in length that play pivotal roles in growth, infiltration, epithelial-to-mesenchymal transition
(EMT), differentiation, angiogenesis, and apoptosis processes of different cancers [9-11]. Despite the limited protein coding ability, they are able to modulate cancer cell behavior by chromatin modification and posttranscriptional regulation [12, 13]. LncRNA growth arrest-specific transcript 5 (GAS5), lowly expressed in many tumors, has been identified as a biomarker of multiple cancers, including non-small-cell lung cancer, bladder cancer, and osteosarcoma [14-16]. For instance, GAS5 acts as competing endogenous RNA (ceRNA) to repress cell proliferation by upregulating hZIP1 expression via sponging miR-223 in clear cell renal cell carcinoma [17]. Consistently, the upregulation of GAS5 suppressed tumor angiogenesis, metastasis, and invasion of colorectal cancer through $\mathrm{Wnt} / \beta$-catenin signaling pathway [18]. On the contrary, deficiency of GAS5 facilitated ovarian cancer cell progression by competing miR-196-5p and regulating HOXA5 [19]. Therefore, the regulatory effect of GAS5 on GC requires further investigation.

MicroRNAs (miRNAs), small non-coding RNAs with 19-25 nucleotides, function as oncogenes or tumor suppres- 
sors to modulate tumorigenesis and metastasis [20]. They are essential regulators of multiple physiological and pathological processes, including proliferation, migration, inflammation, differentiation, and apoptosis, by targeting messenger RNA (mRNA) and resulting in mRNA degradation and protein translation inhibition [21-23]. Aberrant expression of miRNA is involved in the pathogenesis of many diseases, including cancer. For example, miR-18a was capable of reactivating the Epstein-Barr virus (EBV) following defective DNA damage response to improve genomic instability in EBV-associated lymphomas [24]. In addition, miR-18a was upregulated in human glioblastoma tumor tissues and cell lines, and the abundance of miR-18a promoted cell cycle, proliferation, migration, and invasion of glioblastoma [25]. Conversely, Hua Wang et al. demonstrated that the downregulation of miR-18a accelerated the progression of hyaluronate treated human corneal epithelial cells by elevating CTGF expression [26]. Therefore, elucidation of the role of miR-18a in GC cell progression is in need urgently.

In this study, we demonstrated that GAS5 enhanced the killing effect of NK cells by sponging miR-18a in GC. We found GAS5 was downregulated while miR-18a was upregulated in primary NK cells from GC patients. Moreover, the GAS5 knockdown attenuated the killing effect of activated NK cells. Those findings provided promising targets for NK cell-based antitumor therapies.

\section{Patients and methods}

Isolation of NK cells. A total of $24 \mathrm{GC}$ patients and 12 healthy donors were recruited from the First Affiliated Hospital of Zhengzhou University. All those volunteers have signed informed consent and our protocols were approved by the Ethics Committee of the First Affiliated Hospital of Zhengzhou University. Peripheral blood mononuclear cells (PBMCs) were isolated from those GC patients and healthy volunteers using the Human PBMCs separation kit (Solarbio, Beijing, China). Afterward, primary NK cells were extracted and purified from PBMCs using magnetic-activated cell sorting human NK cell isolation kit (Sangon Biotech, Shanghai, China) following the manufacturer's instruction.

Cell culture. GC cell line MGC-803 was purchased from the Cell Research Institute of the Chinese Academy of Sciences (Shanghai, China), human NK cell line NK-92 was purchased from the American Type Culture Collection (ATCC, Manassas, VA, USA). MGC-803 cells were cultured in DMEM medium (Gibco, Grand Island, NY, USA) containing 10\% FBS and $0.1 \%$ penicillin and streptomycin (Invitrogen, CA, USA) in $5 \% \mathrm{CO}_{2}$ incubator at $37^{\circ} \mathrm{C}$. Primary NK cells and NK92 cells were maintained in RPMI-1640 medium containing $10 \%$ FBS, $0.1 \%$ penicillin and streptomycin, and $2 \mathrm{mM}$ L-glutamine. Primary NK cells and NK92 cells were treated with IL-2 $(20 \mathrm{ng} / \mathrm{ml})$ for $24 \mathrm{~h}$ for the activation of NK cells.

Cell transfection. The miR-18a mimic (miR-18a), miR-18a inhibitor (anti-miR-18a), miRNA negative control
(miR-NC), and miRNA negative control inhibitor (antimiR-NC) were purchased from RIBOBIO (Guangzhou, China). Small interfering RNA (siRNA) targeting GAS5 (si-GAS5), negative control (si-NC), GAS5 overexpression vector (GAS5) were synthesized by Genepharma (Shanghai, China). Those vectors were transfected in NK92 cells for $24 \mathrm{~h}$ using Lipofectamine 2000 (Invitrogen).

Quantitative real time polymerase chain reaction (qRT-PCR). Primary NK cells and NK92 cells were incubated with TRIzol reagent (Invitrogen) to extract total RNA. Complementary DNA was synthesized using the PrimeScript RT reagent kit (TaKaRa, Dalian, China). qRT-PCR was conducted to measure GAS5 and miR-18a by SYBR green (Applied Biosystems, Foster City, CA, USA). The primers for GAS5, miR-18a, and GAPDH were listed as follows: GAS5, (Forward, 5'-AGCTGGAAGTTGAAATGG-3'; Reverse, 5'-CAAGCCGACTCTCCATACC-3'); miR-18a, (Forward, 5'-GGTAAGGTGCATCTAGTG-3'; Reverse, 5'-GACTGTTCCTCTCTTCCTC-3'); GAPDH, (Forward, 5'-CCCACTCCTCCACCTTTGAC-3'; Reverse, 5'-GGATCTCGCTCCTGGAAGATG-3').

Cytotoxicity assay. Cell cytotoxicity was evaluated using the LDH assay according to the manufacturer's instruction. Briefly, transfected NK cells were co-incubated with MGC-803 at effector cell/target cell (E:T) ratios of 5:1 for $6 \mathrm{~h}$. Then, the supernatant was collected and cytotoxicity of NK cells against GC cell was measured using the LDH Activity Assay kit (Jiancheng Bioengineering, Nanjing, China).

ELISA. Primary NK cells and NK92 cells (5000 cells/well) were seeded into 96-well plate for $4 \mathrm{~h}$. Then, the cells were centrifuged and the supernatants were collected. The secretion of IFN- $\gamma$ and TNF- $\alpha$ was detected using human IFN- $\gamma$ ELISA kit (Abcam) or human TNF- $\alpha$ ELISA kit (Abcam).

Luciferase reporter assay. Wild type and mutant type luciferase vectors (GAS5-WT and GAS5-MUT) were constructed and co-transfected with miR-18a mimics or miR-NC into NK92 cells using Lipofectamine 2000 transfection reagent. Afterward, luciferase activities were determined by the dual-luciferase assay kit (Promega, Madison, WI, USA).

RNA immunoprecipitation (RIP). RIP assay was conducted using the Magna RIP ${ }^{\mathrm{m} w}$ RNA Binding Protein Immunoprecipitation Kit (Millipore). Briefly, NK cells were lysed by RIP lysis buffer containing RNase inhibitor and protease inhibitor. The lysate of NK cells was incubated with RIP buffer containing magnetic beads conjugated with human anti-Agol or negative control IgG. Next, RNA immunoprecipitated complex was purified using RNase-free DNase I (Thermo Fisher Scientific).

RNA pull-down assay. To further confirm the interaction between GAS5 and miR-18a, RNA pull-down assay was conducted. In brief, NK92 cells were transfected with biotinylated (bio)-miR-18a-WT or miR-18a-MUT (50 nM) using Lipofectamine RNAiMax Reagent (Thermo Fisher Scientific, Waltham, MA, USA), followed by the transfection for 48 
h. Treated NK92 cells were collected and ultrasound, then, the remaining cell lysates were maintained with Magnetic Beads (Thermo Fisher Scientific) for $2 \mathrm{~h}$ at $4^{\circ} \mathrm{C}$, followed by analysis with the RNeasy Mini Kit (Qiagen, Hilden, Germany). Finally, the RNA enrichment was detected by using the RT-qPCR assay.

Statistical analysis. Our data were presented as the means \pm standard deviation (SD) and statistical analyses were carried out using SPSS 13.0 software (Chicago, IL) and GraphPad Prism 7 (GraphPad Inc., San Diego, CA, USA). Student's t-test or ANOVA was utilized to compare between two or more groups. A p-value $<0.05$ was considered as statistically significant.

\section{Results}

Downregulation of GAS5 in NK cells of patients with GC. GAS5 expression in primary NK cells isolated from 24 GC patients and 12 healthy controls was measured by qRT-PCR to investigate the function of GAS5 in NK cells. Also, demographic and clinicopathological data of registered gastric cancer patients and healthy controls are presented in Table 1. As illustrated in Figure 1A, GAS5 was downregulated dramatically in primary NK cells from 24 GC patients compared with 12 healthy controls group. More importantly, the cytotoxicity was reduced distinctly after the co-incuba-
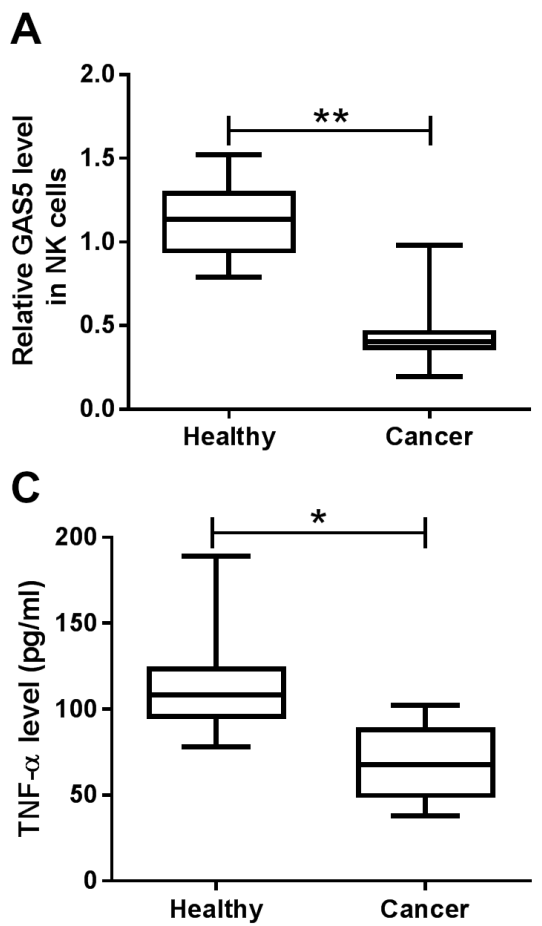

tion of NK cells with MGC-803 cells compared with the healthy controls group (Figure 1B). Moreover, the phenotype of NK cells is displayed in Supplementary Figure S1A. And the percentage of NK cells screened is shown in Supplementary Figures S1B and S1C. Furthermore, compared with the healthy controls group, the levels of IFN- $\gamma$ and TNF- $\alpha$ were downregulated in primary NK cells from 24 GC patients (Figures 1C, 1D). Those results implicated that GAS5 might be associated with NK cell cytotoxicity against GC cells positively.

Table 1. Demographic parameters and clinicopathologic information of patients and healthy donors who participated in the present study.

\begin{tabular}{llcccc}
\hline Assessable & Nale & $\begin{array}{c}\text { No. of } \\
\text { patients }\end{array}$ & $\%$ & $\begin{array}{c}\text { No. of } \\
\text { healthy } \\
\text { donors }\end{array}$ & $\%$ \\
\hline \multirow{2}{*}{ Gender } & Memale & 15 & 62.5 & 8 & 66.7 \\
& $\geq 55$ & 9 & 37.5 & 4 & 33.3 \\
Age (years) & $<55$ & 14 & 58.3 & 7 & 58.3 \\
& I-II & 10 & 41.7 & 5 & 41.7 \\
TNM stage & III-IV & 17 & 70.8 & & \\
\multirow{2}{*}{ Tumor size } & $\geq 5 \mathrm{~cm}$ & 7 & 29.2 & & \\
& $<5 \mathrm{~cm}$ & 16 & 66.7 & & \\
\multirow{2}{*}{ Differentiation } & Well to moderate & 8 & 33.3 & & \\
& Poor & 8 & 66.7 & & \\
\hline
\end{tabular}

B

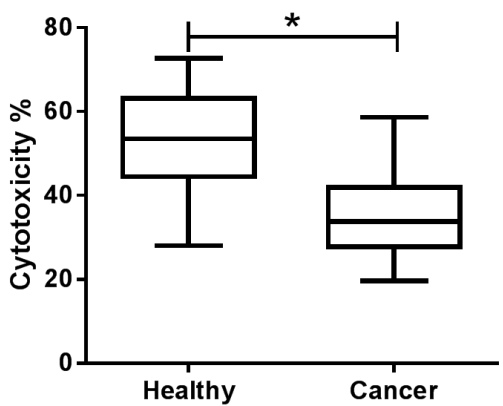

D

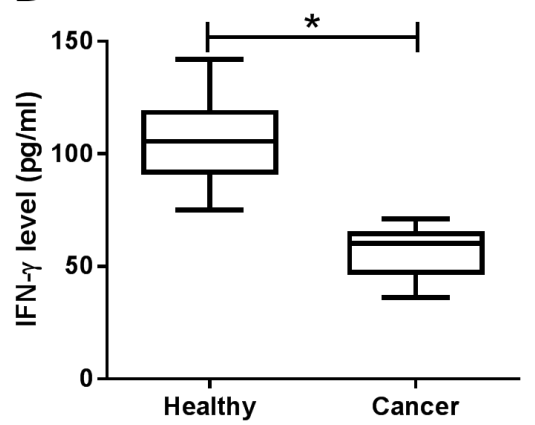

Figure 1. GAS5 was downregulated in NK cells of patients with GC. NK cells were obtained through isolation of peripheral blood from patients with GC $(n=24)$ and healthy controls $(n=12)$. A) Relative GAS5 level in primary NK cells isolated from GC patients and healthy controls was measured by qRT-PCR. B) Primary NK cells were co-incubated with MGC-803 cells to evaluate cytotoxicity using lactate dehydrogenase (LDH) cytotoxicity assay. C, D) The concentration of IFN $-\gamma$ and TNF- $\alpha$ in healthy and cancer derived NK cells was measured using ELISA. ${ }^{*} \mathbf{p}<0.05$ 
Upregulation of GAS5 in activated NK cells. Afterward, primary NK cells from GC patients and human NK cell line NK92 were stimulated with IL-2 to assess the effect of GAS5 on activated NK cells. We observed that the secretion of IFN- $\gamma$ was increased apparently in primary NK cells and NK92 cells treated with IL-2 compared with the control group (Figures 2A, 2B). Consistently, an obvious enhancement of TNF-a expression was discovered in NK cells activated by IL-2 in comparison with the untreated group, indicating the killing effect was promoted after activation of NK cells (Figures 2C, 2D). In addition, GAS5 expression was elevated in primary NK cells and NK92 cells after IL-2 treatment (Figures 2E, 2F). Collectively, GAS5 might be involved in IL-2-induced cytotoxicity activities of NK cells.
GAS5 knockdown suppresses the killing effect of NK cells. To further explore the regulatory effect of GAS5 on NK cells and primary NK cells, NK92 cells and primary NK cells were treated with IL-2 for $24 \mathrm{~h}$ and transfected with si-GAS5 (si-GAS5-1 and si-GAS5-2) or si-NC. As shown in Figure 3A, the GAS5 expression was elevated after IL-2 treatment and repressed after GAS5 silencing. In addition, the abundance of GAS5 enhanced the secretion of IFN- $\gamma$ and TNF- $\alpha$ in the supernatant of NK92 cells or primary NK cells. However, the GAS5 knockdown attenuated the promotive effect of IL-2 on IFN- $\gamma$ and TNF- $\alpha$ secretion (Figures $3 \mathrm{~B}, 3 \mathrm{C}$ ). Then, the killing effect of NK92 cells or primary NK cells was examined by the LDH cytotoxicity assay. As expected, after the co-incubation of NK92 cells or primary NK cells, the cytotoxicity was
A
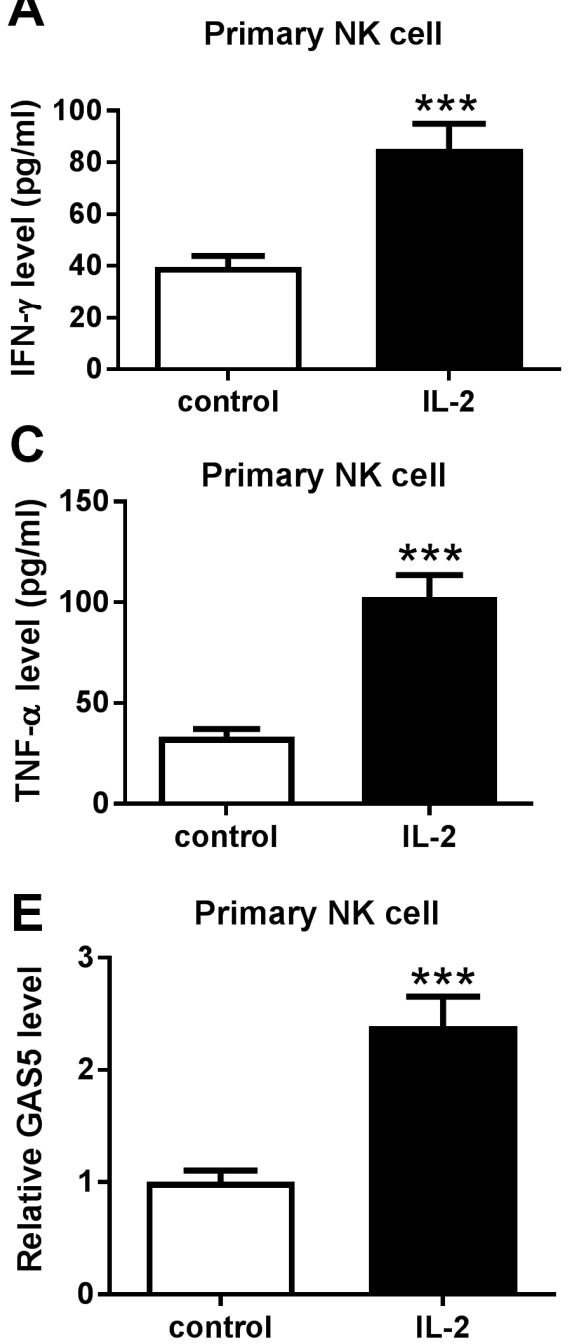

B
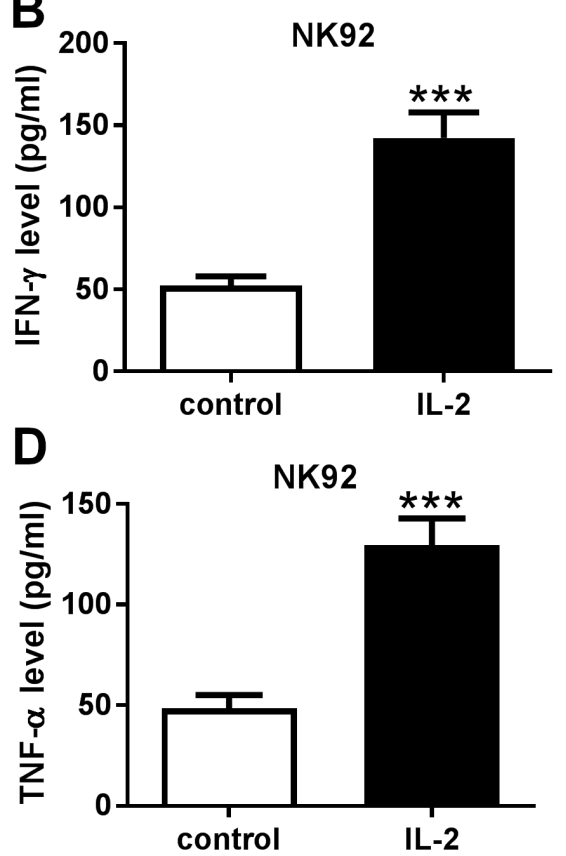

$\mathbf{F}$

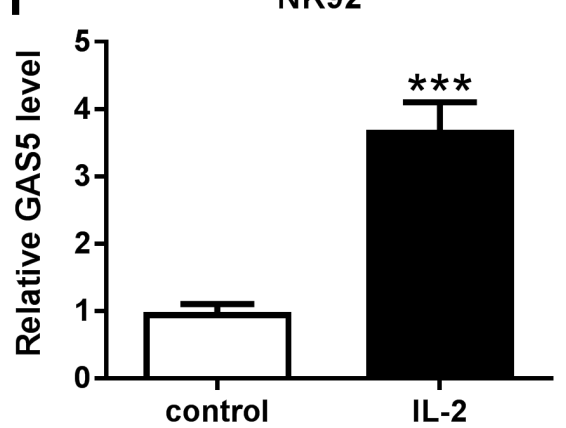

Figure 2. GAS5 was upregulated in activated NK cells. Primary NK cells from GC patients and human NK cell line NK92 were treated with IL-2 (20 ng/ $\mathrm{ml}$ ) for $\mathbf{2 4} \mathrm{h}$ to activate NK cells. Untreated cells were used as the control group. A, B) The secretions of IFN- $\gamma$ in primary NK cells (A) and NK92 cells (B) after IL-2 treatment compared with the control group examined by ELISA. C, D) The secretions of TNF- $a$ in primary NK cells (C) and NK92 cells (D) after IL-2 treatment compared with the control group. E, F) Relative GAS5 level in primary NK cells (E) and NK92 cells (F) after IL-2 treatment compared with the control group. ${ }^{*} \mathrm{p}<0.05$ 
A
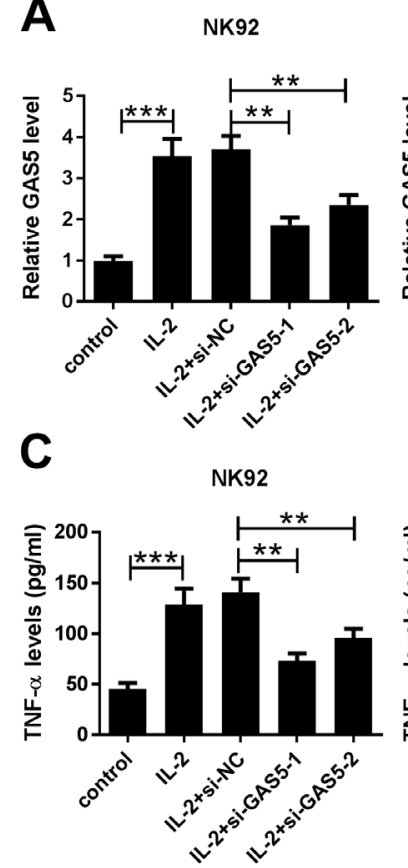
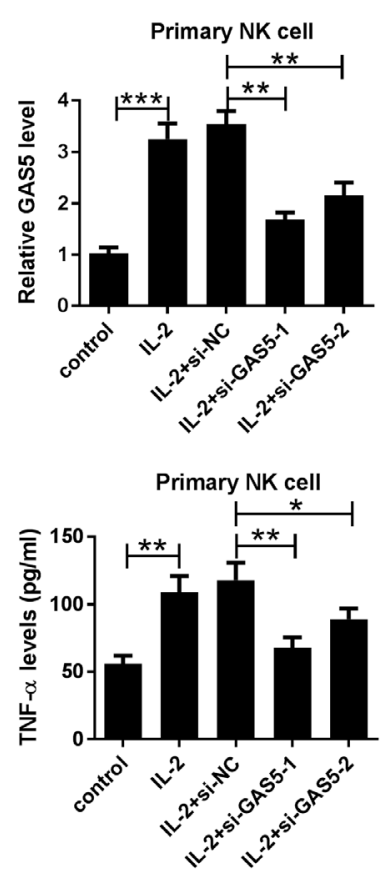

B
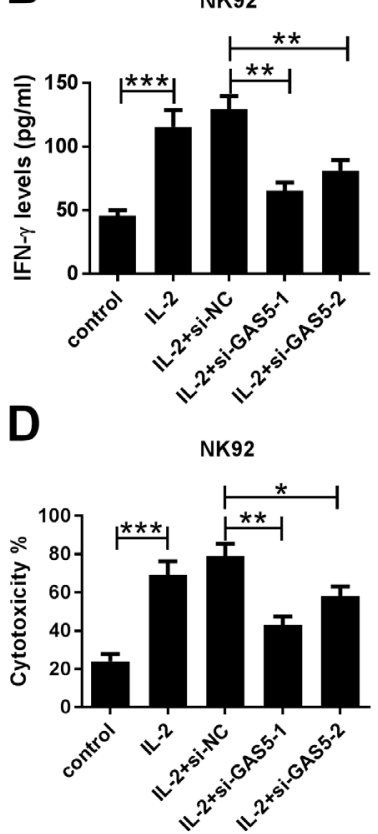

Primary NK cell
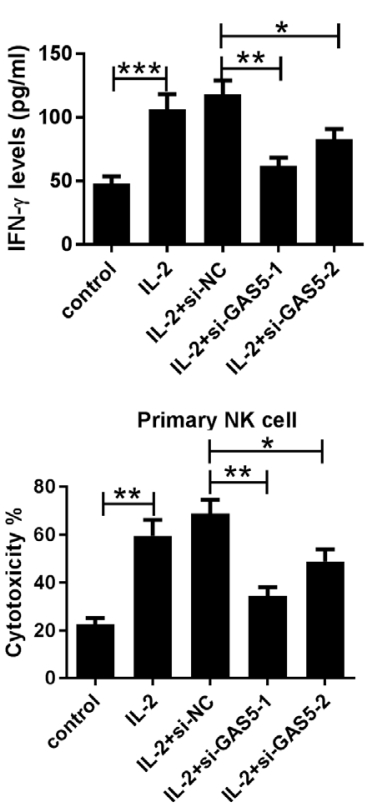

Figure 3. GAS5 knockdown attenuated the killing effect of activated NK cells. NK92 cells and primary NK cells were treated with IL-2 (20 ng/ml) for 24 $h$ and transfected with si-GAS5 and si-NC. A) GAS5 expression in transfected NK92 cells and primary NK cells. B, C) The secretion of IFN- $\gamma(B)$ and TNF- $\alpha(C)$ in NK92 cells and primary NK cells at $24 \mathrm{~h}$ post-transfection. D) NK92 cells or primary NK cells were co-incubated with MGC-803 cells after si-GAS5 and si-NC transfection and cytotoxicity was detected using LDH cytotoxicity assay. ${ }^{*} \mathbf{p}<0.05$

increased in the IL-2 treatment group and decreased in the IL-2 + si-GAS5 group (Figure 3D). Considering these results, we chose si-GAS5-1 for further experiments and concluded that GAS5 knockdown abolished the promotion induced by IL-2 on the killing effect of NK cells or and primary NK cells against GC cells.

GAS5 acts as a molecular sponge of miR-18a. Based on bioinformatics prediction provided by the online database starBase, GAS5 has the potential binding sites to target miR-18a (Figure 4A). Luciferase reporter system and RIP assay were carried out to evaluate the interaction between GAS5 and miR-18a. Typically, wild type GAS5 (GAS5-WT) and mutant type GAS5 (GAS5-MUT) vector were constructed and co-transfected with miR-18a mimics or miR-NC in NK92 cells to establish a luciferase reporter system. As exhibited in Figure 4B, the luciferase activity was reduced remarkably in NK92 cells co-transfected with GAS5-WT and miR-18a whereas that remained unchanged in the GAS5-MUT group. RIP assay result revealed that miR-18a elevated the enrichment of GAS5 in NK92 cells compared with the miR-NC group (Figure 4C). Furthermore, to further validate the binding between GAS5 and miR-18a, RNA pull-down assay was performed. Data showed that folded enrichment of GAS5 was distinctly upregulated in NK92 cells transfected with bio-miR-18a-WT, compared with NK92 cells transfected with bio-miR-18a-MUT and bio-NC (Figure 4D). Moreover, the expression of miR-18a was inhibited by GAS5 and enhanced after GAS5 silencing (Figure 4E). These findings indicated that GAS5 could directly regulate miR-18a in NK92 cells.

Suppression of miR-18a by activating NK cells. We measured the expression of miR-18a in primary NK cells isolated from GC patients and healthy controls by qRT-PCR to investigate the role of miR-18a. We noticed that miR-18a expression was upregulated in NK cells from cancer patients compared with healthy controls (Figure 5A). Additionally, the expression of miR-18a was suppressed in both primary NK cells and NK92 cells treated with IL-2 in comparison with the untreated group (Figures 5B, 5C). Taking together, activation of NK cells by IL-2 suppresses miR-18a expression.

GAS5 promotes NK cell cytotoxicity by regulating miR-18a. NK92 cells and primary NK cells were treated with IL-2 for $24 \mathrm{~h}$ and transfected with si-GAS5, anti-miR-18a, anti-miR-NC, and si-NC to clarify the influence of GAS5/ miR-18a axis on the killing effect of NK cells. As illustrated in Figure 6A, the expression of miR-18a was decreased by IL-2 treatment and increased after GAS5 silencing; however, miR-18a inhibitor reversed GAS5 silencing mediated the promotion on miR-18a expression. Meanwhile, miR-18a inhibitor restored GAS5 silencing induced suppressive effects on the secretion of IFN- $\gamma$ and TNF- $\alpha$ in activated NK cells and primary NK cells (Figures 6B, 6C). Furthermore, the $\mathrm{LDH}$ cytotoxicity assay result indicated that cytotoxicity was downregulated after GAS5 silencing and upregulated by 
A

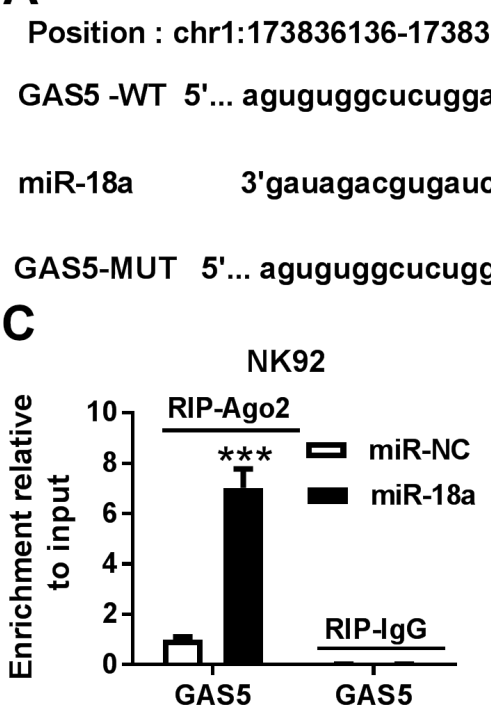

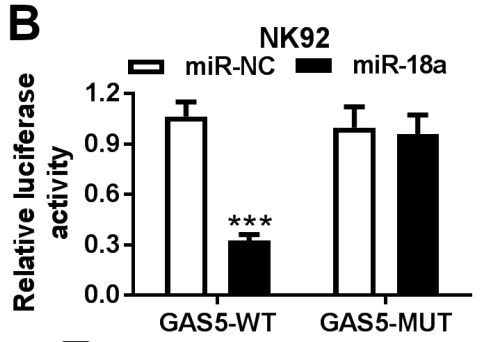
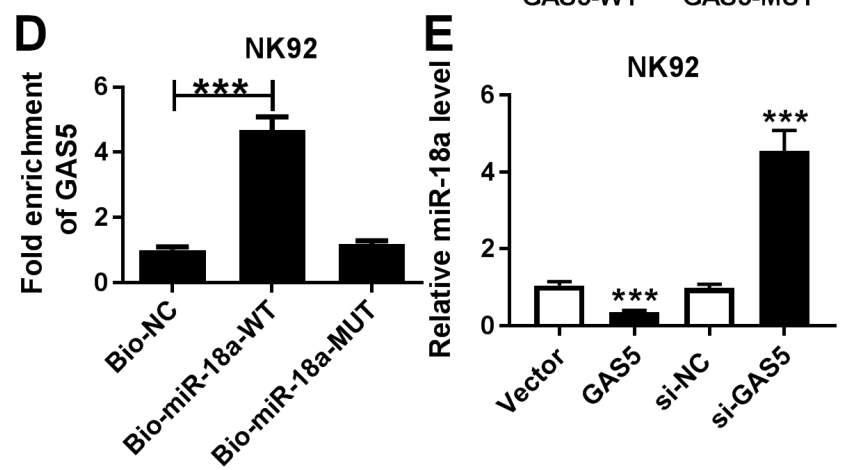

Figure 4. The interaction between GAS5 and miR-18a. A) The putative binding sites of GAS5 and miR-18a searched by starBase. B) Luciferase activity of NK92 cells co-transfected with GAS5-WT or GAS5-MUT and miR-18a mimics or miR-NC. C) The enrichment of GAS5 in NK92 cells transfected with miR-18a and miR-NC evaluated by RIP assay. D) RNA pull-down assay was executed in NK92 cells extracts to validate that GAS5 could directly bind to miR-18a. E) The expression of miR-18a in NK92 cells transfected with GAS5, si-GAS5, si-NC, and vector. * $\mathrm{p}<0.05$
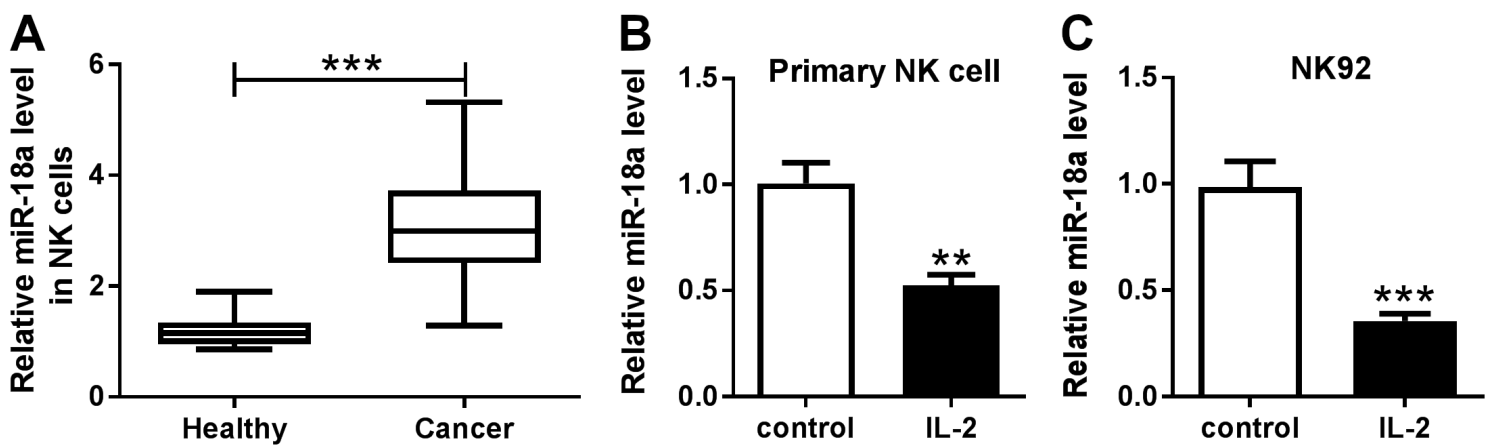

Figure 5. The expression of miR-18a was inhibited by IL-2. A) The expression of miR-18a in primary NK cells isolated from GC patients and healthy controls. B, C) The expression of miR-18a in primary NK cells (B) and NK92 cells (C) treated with IL-2. ${ }^{*} \mathrm{p}<0.05$

miR-18a inhibitor in activated NK cells and primary NK cells (Figure 6D). All the data suggesting that GAS5 enhances the killing effect of NK cells and primary NK cells by regulating $\operatorname{miR}-18 \mathrm{a}$.

\section{Discussion}

It is well acknowledged that NK cells play essential roles in the prognosis of tumors since they could induce immune response after tumor treatment [27]. Moreover, NC cells are capable of lysing the target cells by releasing cytotoxic granules containing perforin and granzyme [28]. In addition, accumulating evidence has validated that lncRNAs widely contribute to the regulation of $\mathrm{NK}$ cytotoxicity in many cancer patients by specifically sponging the corresponding miRNA [29]. GAS5, encoded at prostate cancer associated locus 1q25, is widely accepted as a tumor suppressor of many cancer types [30]. For example, overexpression of GAS5 promoted NK cell cytotoxicity by accelerating IFN- $\gamma$ secretion through regulating miR-544/RUNX3 axis whereas GAS5 knockdown significantly inhibited NK cell cytotoxicity in liver cancer [31]. In addition, GAS5 has been reported to suppress proliferation migration, invasion, and EMT of oral squamous cell carcinoma and triple-negative breast cancer by regulating miR-21/PTEN axis and miR-196a-5p, respectively $[32,33]$. However, whether GAS5 regulates NK cell cytotoxicity of GC patients by interacting with the target miRNA is still obscure. 

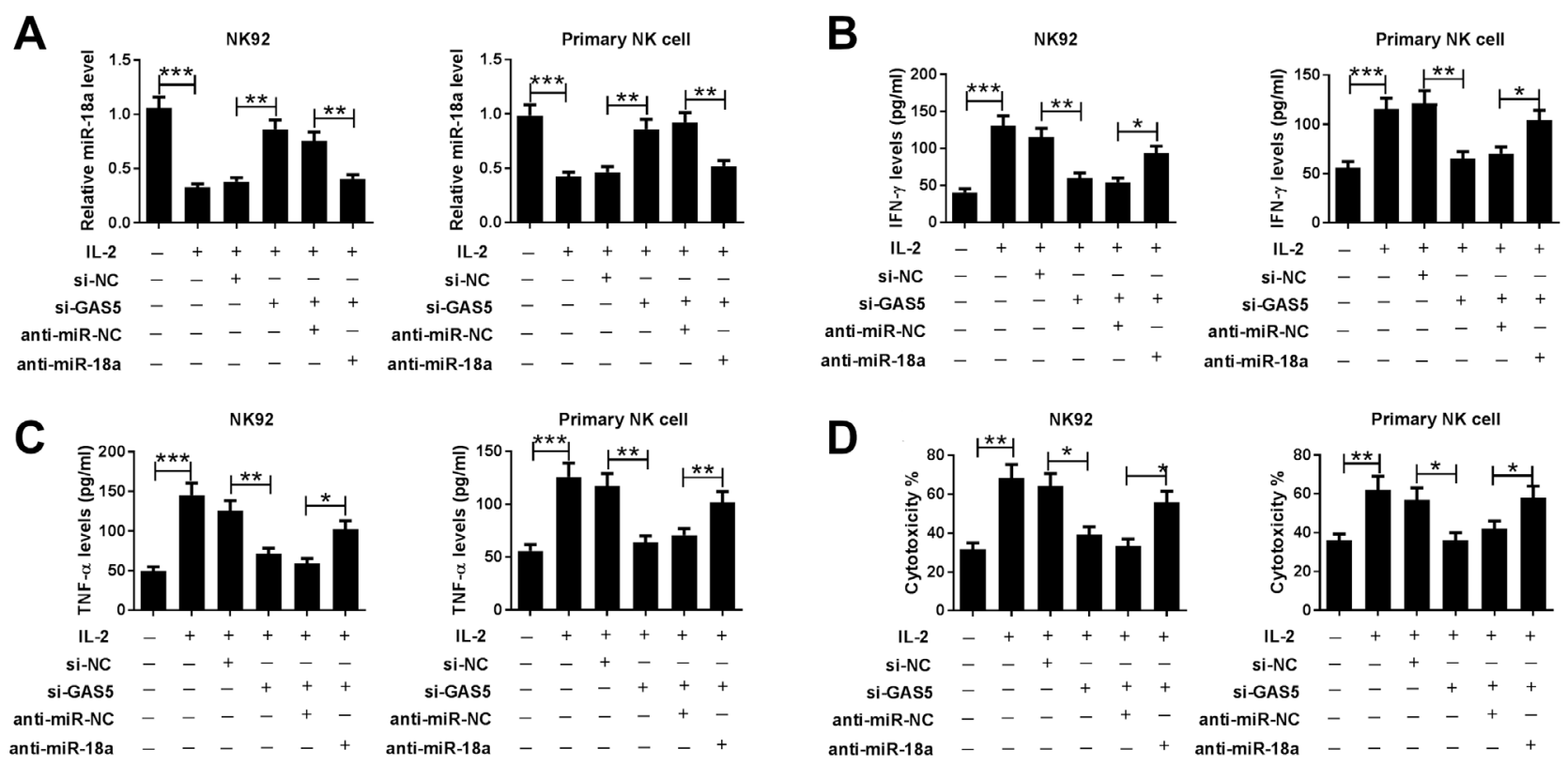

Figure 6. GAS5 enhanced the killing effect of NK cell cytotoxicity by regulating miR-18a. NK92 cells and primary NK cells were treated with IL-2 for $24 \mathrm{~h}$ and transfected with si-GAS5, anti-miR-18a, anti-miR-NC and si-NC. A) The expression of miR-18a in transfected NK92 cells and primary NK cells. B, C) The secretion of IFN- $\gamma$ (B) and TNF- $\alpha$ (C) in NK92 cells and primary NK cells at 24 h post-transfection. D) NK92 cells and primary NK cells were co-incubated with MGC-803 cells after si-GAS5, anti-miR-18a, anti-miR-NC, and si-NC transfection, and cytotoxicity was detected using LDH cytotoxicity assay. ${ }^{*} \mathrm{p}<\mathbf{0 . 0 5}$

Growing evidence has identified that lncRNAs exert their function by acting as molecular sponges of the specific miRNA [34]. According to online database prediction searched by starBase, GAS5 has the potential binding sites to bind to miR-18a. Recently, miR-18a has been clarified as prognostic biomarker of a variety of cancers, like renal cell carcinoma, esophageal cancer, and pediatric acute lymphoblastic leukemia $[35,36]$. Zhang et al. reported that miR-18a exerts its oncogenic function by regulating the PTEN-PI3KAKTmTOR signaling axis to improve Cyclin D1 expression and further accelerate cell cycle progression of esophageal squamous cell carcinoma cells [37]. Likewise, miR-18a was found to upregulate PIAS3 expression at the post-transcriptional level to facilitate malignant mesothelioma cell growth [38]. Besides, the deficiency of miR-18a was discovered to improve cisplatin sensitivity to non-small cell lung cancer cells by targeting PTEN [39]. Thus, understanding the regulatory effect of the GAS/miR-18a axis in GC is of great significance clinically.

We assumed that GAS5 could alter the killing effect of NK cells by interacting with the target miR-18a. Based on the assumption, we detected the expression of GAS5 and miR-18a by qRT-PCR and discovered that GAS5 was downregulated whereas miR-18a was upregulated in primary NK cells isolated from GC patients, implying that GAS5 might inversely regulate miR-18a. In addition, enhanced expression of GAS5 was observed following the activation of NK cells by IL-2. However, GAS5 silencing hindered the killing effect of NK cells. Luciferase reporter and RIP assay further confirmed the interaction between GAS5 and miR-18a. More importantly, miR-18a inhibitor reversed GAS5 silencing induced suppressive effects on the cytotoxicity of activated NK cells.

In conclusion, we firstly clarified the effect of the GAS5/ miR-18a axis on the cytotoxicity of NK cells against GC. Activation of NK cells by IL- 2 enhanced IFN- $\gamma$, TNF- $\alpha$ secretion, GAS5 expression, and cytotoxicity of NK cells by regulating miR-18a. Our study represented potential targets for GC treatment.

Supplementary information is available in the online version of the paper.

\section{References}

[1] BADR EL-DIN NK, ABDEL FATTAH SM, PAN D, TOLENTINO L, GHONEUM M. Chemopreventive Activity of MGN-3/Biobran Against Chemical Induction of Glandular Stomach Carcinogenesis in Rats and Its Apoptotic Effect in Gastric Cancer Cells. Integr Cancer Ther 2016; 15: NP26NP34. https://doi.org/10.1177/1534735416642287

[2] YAMAGUCHI T, FUSHIDA S, YAMAMOTO Y, TSUKADA T, KINOSHITA J et al. Tumor-associated macrophages of the M2 phenotype contribute to progression in gastric cancer with peritoneal dissemination. Gastric Cancer 2016; 19:1052-1065. https://doi.org/10.1007/s10120-015-0579-8 
[3] BECKER PSA, SUCK G, NOWAKOWSKA P, ULLRICH E, SEIFRIED E et al. Selection and expansion of natural killer cells for NK cell-based immunotherapy. Cancer Immunol Immunother 2016; 65: 477-484. https://doi.org/10.1007/ s00262-016-1792-y

[4] LIN M, NIU L, LIANG S, WANG X, LIANG Y et al. Cryoablation combined with allogenic natural killer cell immunotherapy improves the curative effect in patients with advanced hepatocellular cancer. Oncotarget 2017; 8: 8196781977. https://doi.org/10.18632/oncotarget.17804

[5] BAYTAK E, GONG Q, AKMAN B, YUAN H, CHAN WC et al. Whole transcriptome analysis reveals dysregulated oncogenic lncRNAs in natural killer/T-cell lymphoma and establishes MIR155HG as a target of PRDM1. Tumour Biol 2017; 39: 1010428317701648. https://doi. org/10.1177/1010428317701648

[6] KIM R, KAWAI A, WAKISAKA M, FUNAOKA Y, YASUDA $\mathrm{N}$ et al. A potential role for peripheral natural killer cell activity induced by preoperative chemotherapy in breast cancer patients. Cancer Immunol Immunother 2019; 68: 577-585. https://doi.org/10.1007/s00262-019-02305-Z

[7] LIU P, CHEN L, ZHANG H. Natural Killer Cells in Liver Disease and Hepatocellular Carcinoma and the NK CellBased Immunotherapy. J Immunol Res 2018; 2018: 1206737. https://doi.org/10.1155/2018/1206737

[8] YANG C, CHENG H, ZHANG Y, FAN K, LUO G et al. Anergic natural killer cells educated by tumor cells are associated with a poor prognosis in patients with advanced pancreatic ductal adenocarcinoma. Cancer Immunol Immunother 2018; 67: 1815-1823. https://doi.org/10.1007/s00262-0182235-8

[9] CHEN J, WU Z, ZHANG Y. LncRNA SNHG3 promotes cell growth by sponging miR-196a-5p and indicates the poor survival in osteosarcoma. Int J Immunopathol Pharmacol 2019; 33:2058738418820743. https://doi. org/10.1177/2058738418820743

[10] DONG X, HE X, GUAN A, HUANG W, JIA H et al. Long non-coding RNA Hotair promotes gastric cancer progression via miR-217-GPC5 axis. Life Sci 2019; 217: 271-282. https://doi.org/10.1016/j.lfs.2018.12.024

[11] YU J, LIU Y, GONG Z, ZHANG S, LI Y et al. Overexpression long non-coding RNA LINC00673 is associated with poor prognosis and promotes invasion and metastasis in tongue squamous cell carcinoma. Oncotarget 2017; 8: 16621-16632. https://doi.org/10.18632/oncotarget.14200

[12] LI J, LI Y, MENG F, FU L, KONG C. Knockdown of long non-coding RNA linc00511 suppresses proliferation and promotes apoptosis of bladder cancer cells via suppressing Wnt/beta-catenin signaling pathway. Biosci Rep 2018; 38: BSR20171701. https://doi.org/10.1042/BSR20171701

[13] GAO L, WANG X, GUO S, XIAO L, LIANG C et al. LncRNA HOTAIR functions as a competing endogenous RNA to upregulate SIRT1 by sponging miR-34a in diabetic cardiomyopathy. J Cell Physiol 2019; 234: 4944-4958. https://doi. org/10.1002/jcp.27296
[14] WANG M, GUO C, WANG L, LUO G, HUANG C et al. Long noncoding RNA GAS5 promotes bladder cancer cells apoptosis through inhibiting EZH2 transcription. Cell Death Dis 2018; 9: 238. https://doi.org/10.1038/s41419-018-0264-Z

[15] WANG Y, KONG Y. LncRNA GAS5 Represses Osteosarcoma Cells Growth and Metastasis via Sponging MiR203a. Cell Physiol Biochem 2018; 45: 844-855. https://doi. org/10.1159/000487178

[16] LI C, LV Y, SHAO C, CHEN C, ZHANG T et al. Tumor-derived exosomal $\operatorname{lncRNA}$ GAS5 as a biomarker for early-stage non-small-cell lung cancer diagnosis. J Cell Physiol 2019; 234: 20721-20727. https://doi.org/10.1002/jcp.28678

[17] DONG X, KONG C, LIU X, BI J, LI Z et al. GAS5 functions as a ceRNA to regulate hZIP1 expression by sponging miR223 in clear cell renal cell carcinoma. Am J Cancer Res 2018; 8: 1414-1426.

[18] SONG J, SHU H, ZHANG L, XIONG J. Long noncoding RNA GAS5 inhibits angiogenesis and metastasis of colorectal cancer through the Wnt/beta-catenin signaling pathway. J Cell Biochem 2019. https://doi.org/10.1002/jcb.27743

[19] ZHAO H, YU H, ZHENG J, NING N, TANG F et al. Lowlyexpressed lncRNA GAS5 facilitates progression of ovarian cancer through targeting miR-196-5p and thereby regulating HOXA5. Gynecol Oncol 2018; 151: 345-355. https://doi. org/10.1016/j.ygyno.2018.08.032

[20] ZHANG N, ZHANG H, LIU Y, SU P, ZHANG J et al. SREBP1, targeted by miR-18a-5p, modulates epithelial-mesenchymal transition in breast cancer via forming a co-repressor complex with Snail and HDAC1/2. Cell Death Differ 2019; 26: 843-859. https://doi.org/10.1038/s41418-018-0158-8

[21] LU C, PENG K, GUO H, REN X, HU S et al. miR-18a-5p promotes cell invasion and migration of osteosarcoma by directly targeting IRF2. Oncol Lett 2018; 16: 3150-3156. https://doi.org/10.3892/ol.2018.9032

[22] GENG H, GUAN J. MiR-18a-5p inhibits endothelial-mesenchymal transition and cardiac fibrosis through the Notch2 pathway. Biochem Biophys Res Commun 2017; 491: 329336. https://doi.org/10.1016/j.bbrc.2017.07.101

[23] GAO Y, WANG B, LUO H, ZHANG Q, XU M. miR-217 represses TGF-betal-induced airway smooth muscle cell proliferation and migration through targeting ZEB1. Biomed Pharmacother 2018; 108: 27-35. https://doi.org/10.1016/j. biopha.2018.09.030

[24] CAO P, ZHANG M, WANG L, SAI B, TANG J et al. miR-18a reactivates the Epstein-Barr virus through defective DNA damage response and promotes genomic instability in EBVassociated lymphomas. BMC Cancer 2018; 18: 1293. https:// doi.org/10.1186/s12885-018-5205-9

[25] SONG Y, WANG P, ZHAO W, YAO Y, LIU X et al. MiR-18a regulates the proliferation, migration and invasion of human glioblastoma cell by targeting neogenin. Exp Cell Res 2014; 324: 54-64. https://doi.org/10.1016/j.yexcr.2014.03.009

[26] GUO Y, LU X, WANG H. Downregulation of miR-18a induces CTGF and promotes proliferation and migration of sodium hyaluronate treated human corneal epithelial cells. Gene 2016; 591: 129-136. https://doi.org/10.1016/j. gene.2016.07.008 
[27] DENDLE C, GAN PY, POLKINGHORNE KR, NGUI J, STUART RL et al. Natural killer cell function predicts severe infection in kidney transplant recipients. Am J Transplant 2019; 19: 166-177. https://doi.org/10.1111/ajt.14900

[28] TREMBLAY-MCLEAN A, COENRAADS S, KIANI Z, DUPUY FP, BERNARD NF. Expression of ligands for activating natural killer cell receptors on cell lines commonly used to assess natural killer cell function. BMC Immunol 2019; 20: 8 . https://doi.org/10.1186/s12865-018-0272-x

[29] LIU W, LUO M, ZOU L, LIU X, WANG R et al. uNK cell-derived TGF-beta1 regulates the long noncoding RNA MEG3 to control vascular smooth muscle cell migration and apoptosis in spiral artery remodeling. J Cell Biochem 2019; 120: 15997-16007. https://doi.org/10.1002/jcb.28878

[30] GAO J, LIU L, LI G, CAI M, TAN C et al. LncRNA GAS5 confers the radio sensitivity of cervical cancer cells via regulating miR-106b/IER3 axis. Int J Biol Macromol 2019; 126: 994-1001. https://doi.org/10.1016/j.ijbiomac.2018.12.176

[31] FANG P, XIANG L, CHEN W, LI S, HUANG S et al. LncRNA GAS5 enhanced the killing effect of NK cell on liver cancer through regulating miR-544/RUNX3. Innate Immun 2019; 25: 99-109. https://doi.org/10.1177/1753425919827632

[32] ZENG B, LI Y, JIANG F, WEI C, CHEN G et al. LncRNA GAS5 suppresses proliferation, migration, invasion, and epithelial-mesenchymal transition in oral squamous cell carcinoma by regulating the miR-21/PTEN axis. Exp Cell Res 2019; 374: 365-373. https://doi.org/10.1016/j.yexcr.2018.12.014
[33] LI S, ZHOU J, WANG Z, WANG P, GAO X et al. Long noncoding RNA GAS5 suppresses triple negative breast cancer progression through inhibition of proliferation and invasion by competitively binding miR-196a-5p. Biomed Pharmacother 2018; 104: 451-457. https://doi.org/10.1016/j.biopha.2018.05.056

[34] FEI D, ZHANG X, LIU J, TAN L, XING J et al. Long Noncoding RNA FER1L4 Suppresses Tumorigenesis by Regulating the Expression of PTEN Targeting miR-18a-5p in Osteosarcoma. Cell Physiol Biochem 2018; 51: 1364-1375. https:// doi.org/10.1159/000495554

[35] ZHOU L, LI Z, PAN X, LAI Y, QUAN J et al. Identification of miR-18a-5p as an oncogene and prognostic biomarker in RCC. Am J Transl Res 2018; 10: 1874-1886.

[36] MOSAKHANI N, EL MISSIRY M, VAKKILA E, KNUUTILA S, VAKKILA J. Low Expression of miR-18a as a Characteristic of Pediatric Acute Lymphoblastic Leukemia. J Pediatr Hematol Oncol 2017; 39: 585-588. https://doi.org/10.1097/ MPH.0000000000000921

[37] ZHANG W, LEI C, FAN J, WANG J. miR-18a promotes cell proliferation of esophageal squamous cell carcinoma cells by increasing cylin D1 via regulating PTEN-PI3K-AKT-mTOR signaling axis. Biochem Biophys Res Commun 2016; 477: 144-149. https://doi.org/10.1016/j.bbrc.2016.06.034

[38] HE T, MCCOLL K, SAKRE N, CHEN Y, WILDEY G et al. Post-transcriptional regulation of PIAS3 expression by miR18a in malignant mesothelioma. Mol Oncol 2018; 12: $2124-$ 2135. https://doi.org/10.1002/1878-0261.12386

[39] XIAO H, LIU Y, LIANG P, WANG B, TAN H et al. TP53TG1 enhances cisplatin sensitivity of non-small cell lung cancer cells through regulating miR-18a/PTEN axis. Cell Biosci 2018; 8: 23. https://doi.org/10.1186/s13578-018-0221-7 